\title{
Curcumin suppresses cardiac fibroblasts activities by regulating the proliferation and cell cycle via the inhibition of the p38 MAPK/ERK signaling pathway
}

\author{
GUANHUA FANG ${ }^{*}$, SHAOQIN CHEN* , QIUYU HUANG, LIANGWAN CHEN and DONGSHAN LIAO \\ Department of Cardiac Surgery, Fujian Medical University Union Hospital, Fuzhou, Fujian 350001, P.R. China
}

Received December 13, 2017; Accepted May 9, 2018

DOI: $10.3892 / \mathrm{mmr} .2018 .9120$

\begin{abstract}
Cardiac fibrosis is a deleterious effect of many cardiovascular diseases. Previous studies have shown that curcumin has exhibited protective effects on cardiovascular diseases. The aim of the present study was to evaluate the effects of curcumin on the activity of human cardiac fibroblasts (CFs) and to elucidate the underlying mechanisms involved. Human CFs were incubated with or without curcumin $(20 \mu \mathrm{mol} / \mathrm{l})$ and transforming growth factor $\beta 1$ (TGF- $\beta 1 ; 10 \mathrm{ng} / \mathrm{ml}$ ), and the expression of $\alpha$-smooth muscle actin ( $\alpha$-SMA), collagen type I $\alpha$ (COLA)-1 and COLA3 was evaluated using reverse transcription-quantitative polymerase chain reaction and western blot analysis. Cell proliferation was evaluated by Cell Counting Kit- 8 analysis, and phases of the cell cycle were studied by flow cytometry. Western blot analysis was performed to evaluate the expression of cyclin-dependent kinase 1 (CDK1), Cyclin B, phosphorylation (p)-mothers against decapentaplegic homolog 2/3 (p-smad2/3), p-P38, and p-extracellular regulated protein kinases (ERK). Curcumin significantly reduced mRNA and protein levels of $\alpha$-SMA, COLA 1 , and COLA 3 in CFs stimulated with TGF- $\beta 1$. However, in the absence of TGF- $\beta 1$, curcumin did not have any effects on CFs, suggesting that curcumin inhibited TGF- $\beta 1$-mediated $\mathrm{CF}$ activities, including differentiation and collagen deposition. Additionally, curcumin inhibited the proliferation of TGF- $\beta 1$-treated CFs, and promoted G2/M phase cell cycle arrest. Curcumin reduced cell cycle protein expression by inhibiting smad2/3, p38 mitogen-activated protein kinase, and ERK phosphorylation in TGF- $\beta 1$-treated CFs. Thus, these
\end{abstract}

Correspondence to: Professor Dongshan Liao or Professor Liangwan Chen, Department of Cardiac Surgery, Fujian Medical University Union Hospital, 29 Xinquan Road, Fuzhou, Fujian 350001, P.R. China

E-mail: liao890612@qq.com

E-mail: clw1579@tom.com

*Contributed equally

Key words: cardiac fibroblasts, curcumin, p38 mitogen-activated protein kinase/extracellular signal-regulated kinases signaling pathway results indicated that curcumin may be a potential anti-fibrotic drug to treat cardiac fibrosis.

\section{Introduction}

Cardiac fibrosis is a major aspect in the remodeling of diverse cardiovascular diseases, including atherosclerosis, hypertension, arrhythmias, ischemic and dilated cardiomyopathy (1). Excessive cardiac fibrosis can lead to cardiac dysfunction, interstitial remodeling, structural disorder, and eventually progressive heart failure (1). Cardiac fibrosis is characterized by the net accumulation of extracellular matrix in the cardiac interstitium (2), and cardiac fibroblasts (CFs), main effector cells in cardiac fibrosis, play an important role in the formation of cardiac fibrosis (3). Transforming growth factor $\beta 1$ (TGF- $\beta 1$ ) is a potent profibrotic cytokine and initiates and maintains fibrotic responses (4). CFs that are induced by TGF- $\beta 1$ can transform into myofibroblasts that exhibit augmented proliferative, migratory, contractile, and collagen-producing abilities (2,5). A better understanding of the regulation of CFs would help ameliorate the deleterious effects of cardiac fibrosis.

Curcumin (diferuloylmethane) has been reported to exhibit several beneficial properties, including anti-inflammatory, anti-oxidative, anti-proliferative, and anti-apoptotic activities (6). Accumulating evidence has demonstrated preventive effects of curcumin in fibrotic diseases, including oral submucosal (7), liver (8), lung (9), and kidney fibrosis (10). In a previous study, the cardiac protective effect of curcumin was demonstrated in several heart diseases, such as hypertension, myocardial infarction, and diabetic cardiomyopathy (11-13). The protective effects of curcumin on myocardial injury have been reported to be anti-inflammatory, however, the anti-fibrotic properties of curcumin on cardiac fibrosis have yet to be elucidated.

To determine effects of curcumin on CFs, we evaluated the proliferation, cell cycle phase, and collagen deposition in CFs. Our results revealed that curcumin inhibited TGF- $\beta 1$-induced cardiac fibroblast proliferation, differentiation, and collagen production, and may be mediated by inhibiting the Smad and p38 MAPK pathways.

\section{Materials and methods}

Cells and reagents. Normal human CFs were obtained from ScienCell Research Laboratories (San Diego, CA, USA). 
TGF- $\beta 1$ was obtained from R\&D Systems, Inc., (Minneapolis, MN, USA). Antibodies directed against $\alpha$-smooth muscle actin ( $\alpha$-SMA), collagen type I $\alpha$ (COLA)-1, COLA3, cyclin-dependent kinase 1 (CDK1), cyclin B, phospho-smad2/3 (p-smad2/3), phospho-p38 mitogen-activated protein kinase (p-p38 MAPK), phospho-extracellular regulated protein kinases (p-ERK), and GAPDH were purchased from Bioss Antibodies (Beijing, China).

Cell treatment. CFs were cultured in Dulbecco's modified Eagle's medium (DMEM; Invitrogen; Thermo Fisher Scientific, Inc., Waltham, MA, USA), supplemented with $10 \%$ fetal bovine serum (Hangzhou Sijiqing Biological Engineering Materials Co., Ltd., Hangzhou, China), $100 \mathrm{U} / \mathrm{ml}$ penicillin and $100 \mathrm{mg} / \mathrm{ml}$ streptomycin. Cells were cultured at $37^{\circ} \mathrm{C}$ in a $5 \% \mathrm{CO}_{2}$ atmosphere. CFs were treated with/without TGF- $\beta 1(10 \mathrm{ng} / \mathrm{ml})$ for $24 \mathrm{~h}$ or pretreated with curcumin $(20 \mu \mathrm{mol} / \mathrm{l})$ for $1 \mathrm{~h}$ prior to stimulation with TGF- $\beta 1$.

Proliferation assay. The proliferation of CFs was evaluated by a commercial Cell Counting Kit-8 (CCK-8; Dojindo Molecular Technologies, Inc., Kumamoto, Japan). A total of $3 \times 10^{3}$ CFs were seeded in each well of a 96-well plate and $10 \mu \mathrm{lCCK}-8$ solution was added to each well $\mathrm{w}$ for $1,2,3,4,5,6$, or 7 days. Then, the optical density (at $450 \mathrm{~nm}$ ) was measured using a microplate reader (Thermo Fisher Scientific, Inc.), and the cell viability was calculated.

Cell cycle assay. CFs were seeded in 6-well plates at a density of $4 \times 10^{4}$ cells/well and cultured for $24 \mathrm{~h}$ at $37^{\circ} \mathrm{C}$. Next, CFs were treated with/without TGF- $\beta 1(10 \mathrm{ng} / \mathrm{ml})$ for $24 \mathrm{~h}$ or pretreated with curcumin $(20 \mu \mathrm{mol} / \mathrm{l})$ for $1 \mathrm{~h}$ prior to stimulation with TGF- $\beta 1$. Cells were washed with phosphate-buffered saline (PBS), fixed with $70 \%$ ethanol for $2 \mathrm{~h}$ at $4^{\circ} \mathrm{C}$ and then the cells were incubated for $30 \mathrm{~min}$ with $10 \mathrm{mg} / \mathrm{ml}$ propidium iodide (PI) and $2.5 \mathrm{mg} / \mathrm{ml}$ DNase-free RNase (Beyotime Institute of Biotechnology, Haimen, China) for staining of DNA. A total of $2 \times 10^{4}$ cells were analyzed by flow cytometry on a BD FACSCanto flow cytometer. FlowJo software was used for data analysis (Tree Star, Inc., Ashland, OR, USA).

Reverse transcription-quantitative polymerase chain reaction $(R T-q P C R)$. RNA was extracted from cells by TRIzol reagent (Invitrogen; Thermo Fisher Scientific, Inc.) and reverse transcribed into cDNA by a M-MLV Reverse Transcriptase kit (Promega, Madison, WI, USA). qPCR was performed on an Applied Biosystems 7500 Real-Time PCR system (Thermo Fisher Scientific, Inc.). mRNA levels of $\alpha$-SMA, COLA1, and COLA3 were determined by RT-qPCR using GAPDH as an internal control. Primer sequences are shown in Table I. PCR amplification was carried out by denaturation at $94^{\circ} \mathrm{C}$ for $5 \mathrm{sec}$ followed by 40 cycles of annealing and extension $\left(62^{\circ} \mathrm{C}\right.$ for $40 \mathrm{sec})$. Relative gene expression was determined using the $2^{-\Delta \Delta \mathrm{Cq}}$ method.

Western blot analysis. Total protein was extracted from cells using RIPA buffer (Thermo Fisher Scientific, Inc.) and quantified using the BCA Protein Assay Kit following the manufacturer's instructions (Thermo Fisher Scientific, Inc.). A total of $20 \mu \mathrm{g}$ protein was loaded onto SDS-PAGE gels,
Table I. Primer sequences.

\begin{tabular}{ll}
\hline Gene & \multicolumn{1}{c}{ Primer sequence $\left(5^{\prime}-3^{\prime}\right)$} \\
\hline GAPDH & F: ACATCATCCCTGCCTCTACTG \\
& R: CCTGCTTCACCACCTTCTTG \\
a-SMA & F: TTCCTTCGTGACTACTGCTGAG \\
& R: GAAAGATGGCTGGAAGAG \\
COLA1 & F: GAATATGTATCACCAGACGCAGAAG \\
& R: AGACCA CGAGGACCAGAAGG \\
COLA3 & F: CTGGAGTCGGAGGAATGG \\
& R: GCCAGATGGACCAATAGCA
\end{tabular}

SMA, smooth muscle actin; COLA, collagen type I $\alpha$; F, forward; R, reverse.

proteins were separated by electrophoresis, electrotransferred onto polyvinylidene difluoride (PVDF) membranes (EMD Millipore, Billerica, MA, USA), and blocked for $1 \mathrm{~h}$ at room temperature using tris-buffered saline (TBS)-Tween-20 (TBST) (J\&K Scientific, Ltd., Shanghai, China) containing 5\% non-fat dry milk. Subsequently, membranes were probed overnight at $4^{\circ} \mathrm{C}$ with rabbit polyclonal antibodies (1:500) directed against a-SMA, COLA1, COLA3, Cyclin B, CDK1, p-Smad2/3, p-p38 MAPK, p-ERK, and GAPDH in TBST. After incubation with horseradish peroxidase (HRP)-conjugated secondary antibodies, proteins were visualized using an enhanced chemiluminescence (ECL) substrate kit (Thermo Fisher Scientific, Inc.). GAPDH was used as a loading control.

Statistical analysis. Statistical analyses were carried out using IBM SPSS Statistics software v20.0 (IBM Corp., Armonk, NY, USA). Data are presented as the mean \pm standard deviation of three independent experiments, and compared using the post-hoc test used with analysis of variance with Tukey's post hoc test. $\mathrm{P}<0.05$ was considered to indicate a statistically significant difference.

\section{Results}

Curcumin inhibits TGF- $\beta 1$-induced differentiation of CFs. To determine the effects of curcumin on cardiac fibrosis, we evaluated the level of $\alpha$-SMA, a biomarker of myofibroblast differentiation. As shown in Fig. 1, both gene and protein levels of $\alpha$-SMA in TGF- $\beta 1$-stimulated CFs were significantly increased compared with the controls, and elevated levels of $\alpha$-SMA were reduced in curcumin-treated CFs.

Curcumin reduces collagen deposition of TGF- $\beta 1$-induced $C F s$. To investigate the effects of curcumin on collagen accumulation, we determined the protein expression of collagen I and III, which are major components of extracellular matrix. As shown in Fig. 2, when compared to TGF- $\beta 1$-stimulated $\mathrm{CFs}$, treatment with curcumin significantly reduced protein expression of collagen I and III.

Curcumin inhibits cell proliferation and promotes cell cycle arrest of TGF- $\beta 1$-induced CFs. Excessive proliferation of 

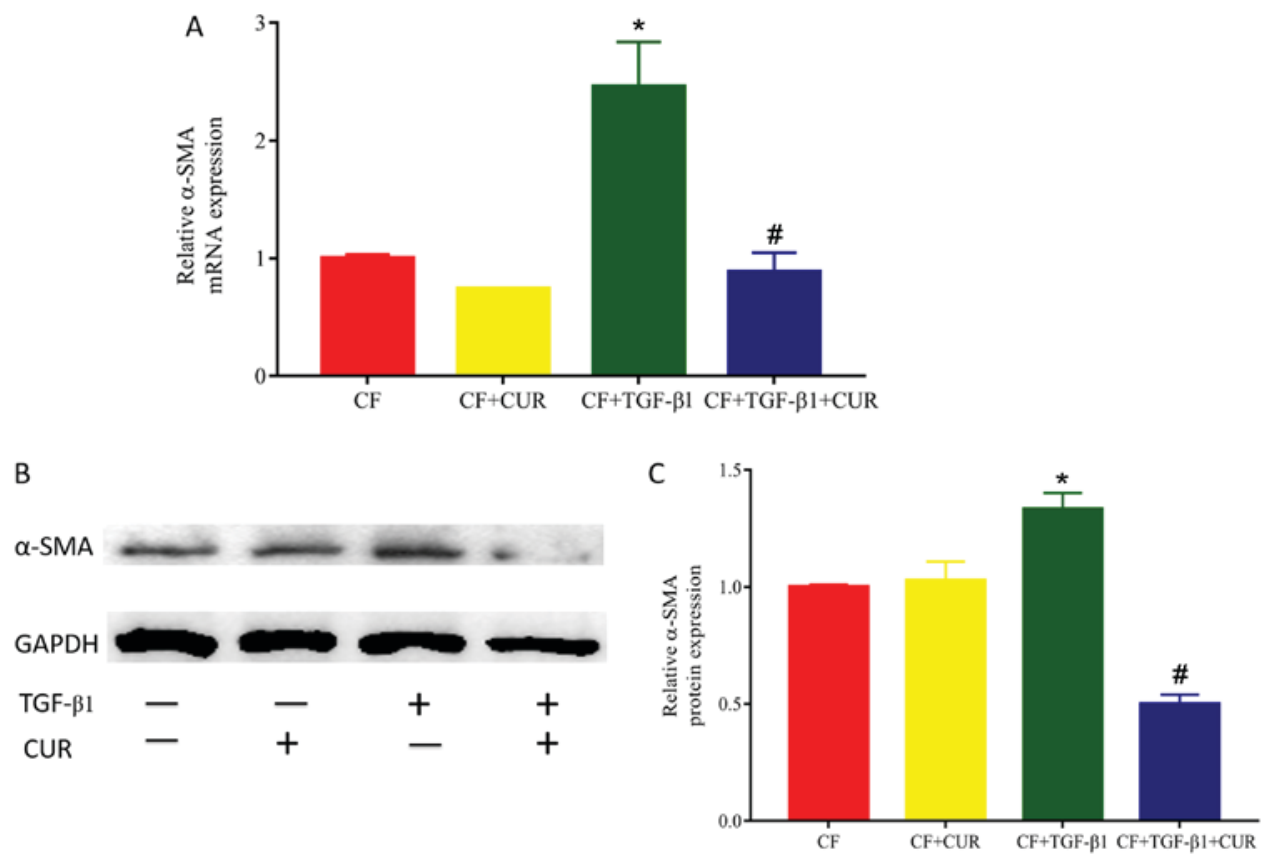

Figure 1. CUR inhibits differentiation in TGF- $\beta 1$-induced CFs. (A) Relative expression of $\alpha$-SMA mRNA. (B) Western blot analysis of $\alpha$-SMA protein. (C) Quantitative analysis of $\alpha$-SMA protein expression. Data are presented as the mean \pm standard deviation of triplicate experiments. "P<0.05 vs. CUR, curcumin; $\mathrm{CF}$ group; ${ }^{\mathrm{P}}<0.05$ vs. $\mathrm{CF}+\mathrm{TGF}-\beta 1$ group. $\mathrm{CFs}$, cardiac fibroblasts; TGF, transforming growth factor; SMA, smooth muscle actin.
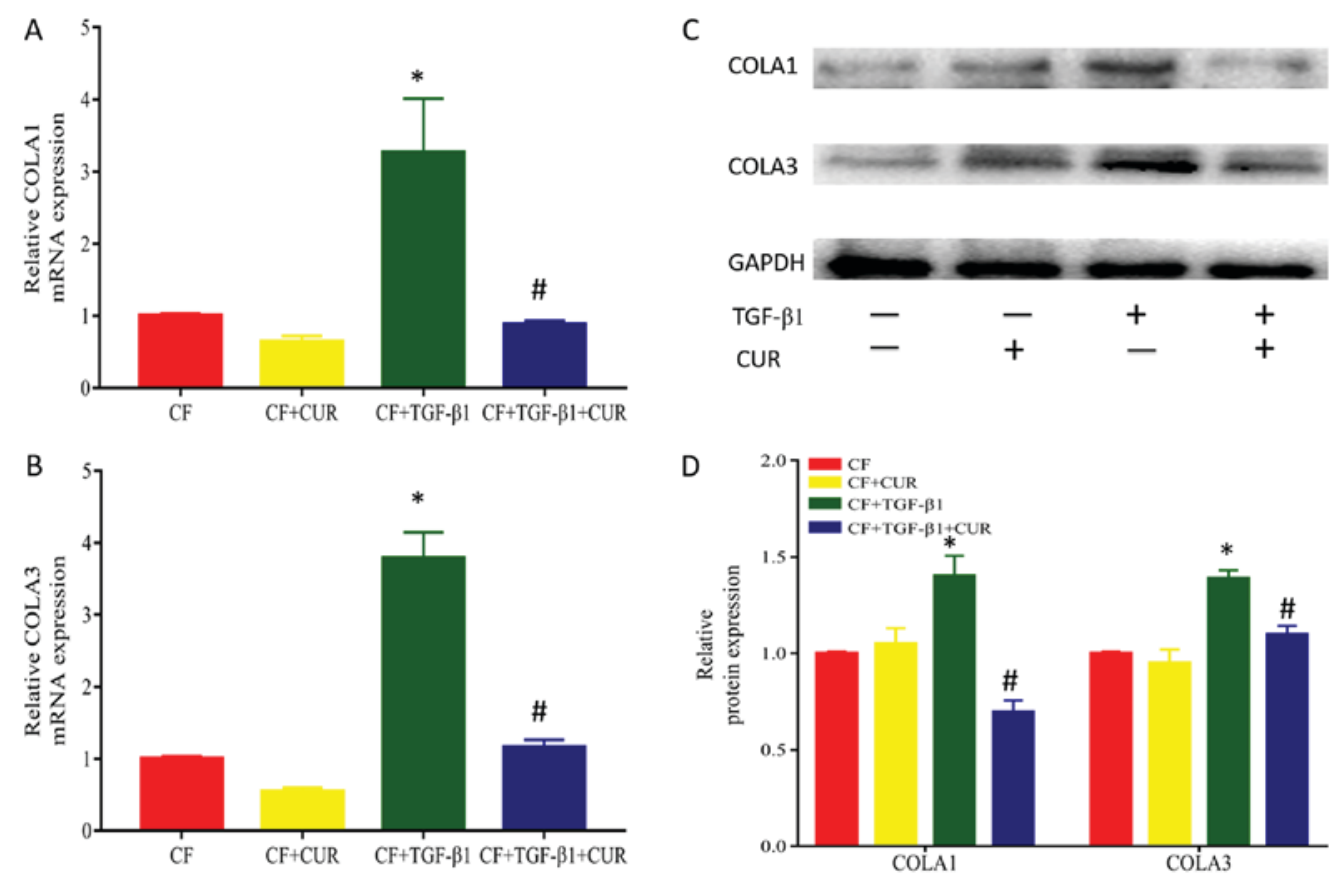

Figure 2. CUR reduces TGF- $\beta 1$-induced collagen deposition of CFs. Relative mRNA expression of (A) COLA1 and (B) COLA3. (C) Western blot analysis of COLA1 and COLA3 expression. (D) Quantitative analysis of COLA1 and COLA3 protein expression. Data are presented as the mean \pm standard deviation of triplicate experiments. "P<0.05 vs. CF group; ${ }^{\#} \mathrm{P}<0.05$ vs. CF + TGF- $\beta 1$ group. CUR, curcumin; TGF, transforming growth factor; CFs, cardiac fibroblasts; COLA, collagen type I $\alpha$.

CFs plays a key role in myocardial fibrosis (14). Herein, we evaluated the proliferation of CFs by using CCK-8 assays. Curcumin pretreatment had no significant effect on the proliferation of CFs. However, CFs displayed increased proliferative abilities after $24 \mathrm{~h}$ of TGF- $\beta 1$ stimulation. These increased proliferative abilities were suppressed by curcumin pretreatment in a dose-dependent manner (Fig. 3A), and were observed from 1 to 7 days after curcumin administration. Flow cytometry was performed to evaluate the effects on the cell cycle phases of CFs. Our findings demonstrated that curcumin significantly decreased the percentage of TGF- $\beta 1$-stimulated CFs in the G0/G1 phase but increased the percentage of cells in the $\mathrm{G} 2 / \mathrm{M}$ phase (Fig. 3C and D), indicating that curcumin causes $\mathrm{G} 2 / \mathrm{M}$ cell cycle arrest. 
A
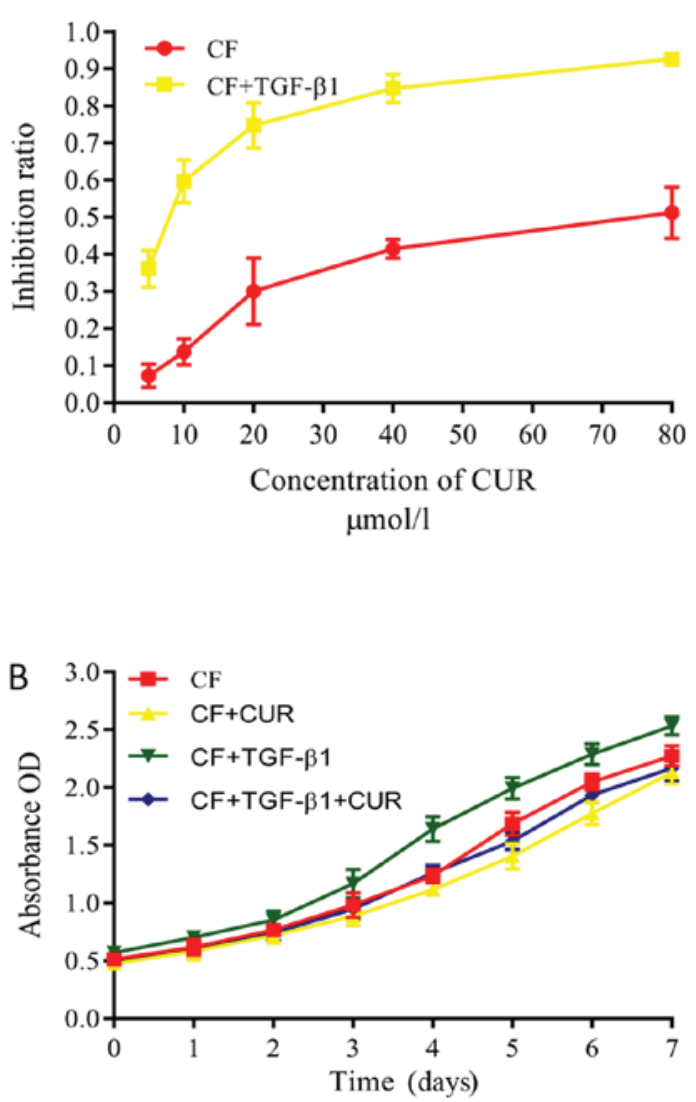

C
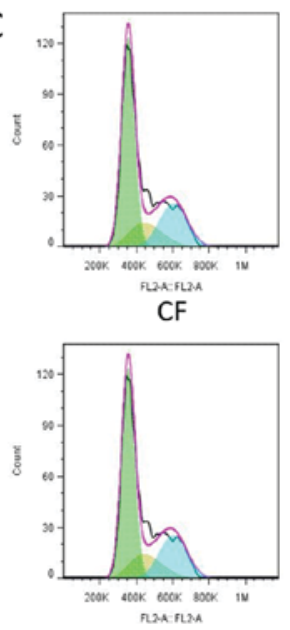

CF+TGF- $\beta 1$

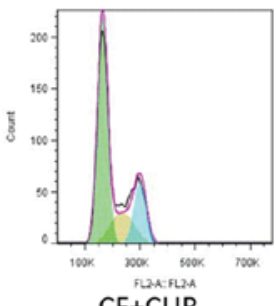

CF+CUR

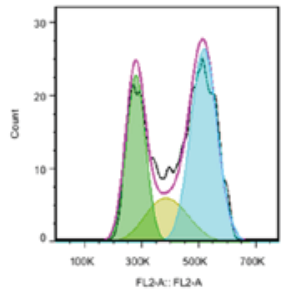

CF+CUR+TGF- $\beta 1$

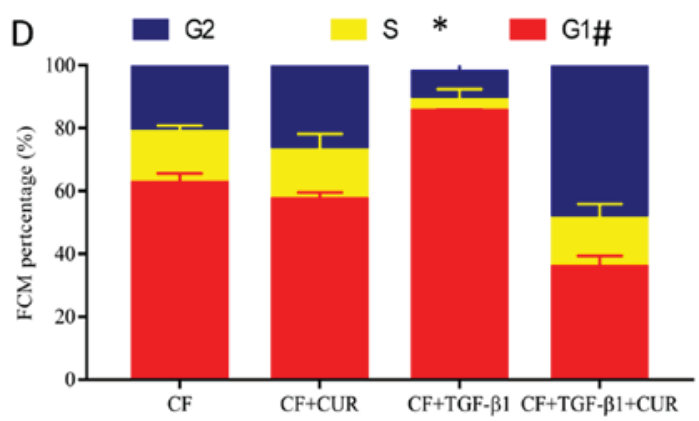

Figure 3. CUR inhibits cell proliferation and promotes the cell-cycle arrest of TGF- $\beta 1$-stimulated CFs. (A) Inhibitory effect of different concentrations of curcumin on the cell proliferation of CFs that were stimulated with or without TGF- $\beta 1$. (B) Inhibitory effect of curcumin on the cell proliferation of CFs stimulated with or without TGF- $\beta 1$ on days 1 to 7. (C) Flow cytometry analysis was performed to (D) evaluate the effects of curcumin on the cell cycle phase of CFs. Data are presented as the mean \pm standard deviation of triplicate experiments. ${ }^{*} \mathrm{P}<0.05$ vs. CF group; ${ }^{*} \mathrm{P}<0.05$ vs. CF + TGF- $\beta 1$ group. CUR, curcumin; TGF, transforming growth factor; $\mathrm{CFs}$, cardiac fibroblasts; OD, optical density.

Curcumin affects cyclin $B$ and CDK1 expression via inhibiting Smad2/3, p38 MAPK, and ERK signaling activation in TGF- $\beta 1$-induced CFs. To further explore the effect of curcumin on cell cycle arrest, we determined the levels of cyclin B and CDK1 protein expression. Our results revealed that the protein expression of cyclin B and CDK1 was elevated after TGF- $\beta 1$ stimulation, and this effect was suppressed by curcumin pretreatment.

Given that TGF- $\beta 1$ is the most important mediator in cardiac fibrosis, we examined proteins involved in TGF- $\beta 1$ signaling in CFs. In the canonical signaling pathway, TGF- $\beta 1$ activates down-stream Smad2/3 pathways to exert its pro-fibrotic effects (14). To determine the effect of curcumin effect on Smad $2 / 3$, we evaluated the protein expression of phosphorylated Smad 2/3 in CFs. As shown in Fig. 4A and D, Smad2/3 phosphorylation was significantly enhanced after TGF- $\beta 1$ stimulation, and this effect was significantly reduced by curcumin.

In addition, it has been described that TGF- $\beta 1$ activates the non-canonical MAPK pathway to modulate profibrotic processes (14). As shown in Fig. 4A-C, TGF- $\beta 1$ did not affect p38 and ERK expression, but enhanced p38 and ERK phosphorylation. Curcumin pretreatment significantly reduced increased phosphorylation of p38 MAPK and ERK. Thus, these findings indicated that curcumin suppressed p38 MAPK and ERK activation in response to TGF- $\beta 1$ in CFs.

\section{Discussion}

Curcumin exhibits promising pharmacological activities and has demonstrated to prevent properties in multiple fibrotic diseases, such as oral submucosal (7), liver (8), lung (9), and kidney fibrosis (10). In the present study, we evaluated the protective effect of curcumin on cardiac fibrosis. Curcumin inhibited TGF- $\beta 1$-induced proliferation, differentiation, and collagen deposition of CFs, and promoted cell apoptosis. The inhibitory effects of curcumin may be caused by inhibition of Smad2/3, p38 MAPK, and ERK signaling pathways. Together, these findings suggested that curcumin may be of potential therapeutic use for the treatment of cardiac fibrosis.

Transformation of cardiac myofibroblasts is a central event of cardiac fibrosis, which is featured by high expression of $\alpha$-SMA $(15,16)$. Collagen I and III are major components of the extracellular matrix, which are excessively synthesized by cardiac myofibroblasts (17). It has been well documented that TGF- $\beta 1$ enhances the proliferative, migratory, and collagen-producing properties of $\mathrm{CFs}$ by inducing myofibroblast differentiation (4). In the present study, we used TGF- $\beta 1$ to stimulate CFs to possibly mimic the 'fibrotic' situation. Herein, pretreatment with curcumin significantly decreased mRNA and protein expression of $\alpha$-SMA, suggesting that curcumin-suppressed TGF- $\beta 1$ induced cardiac fibroblast 
A
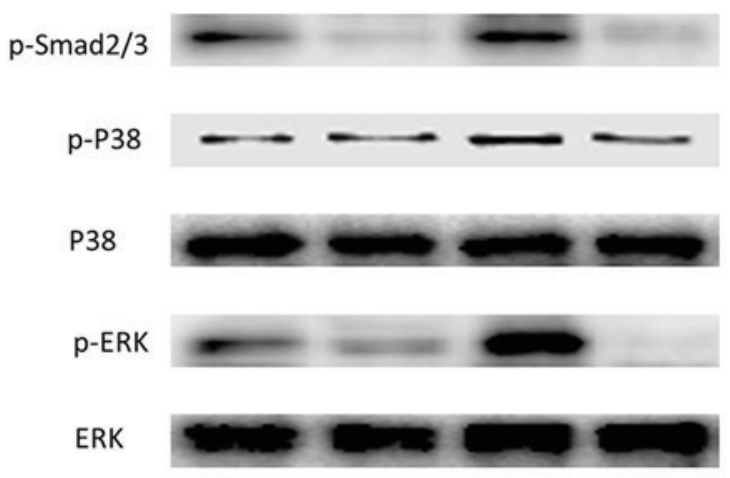

Cyclin B

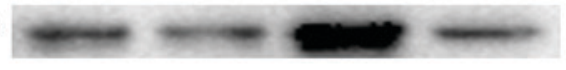

CDK1
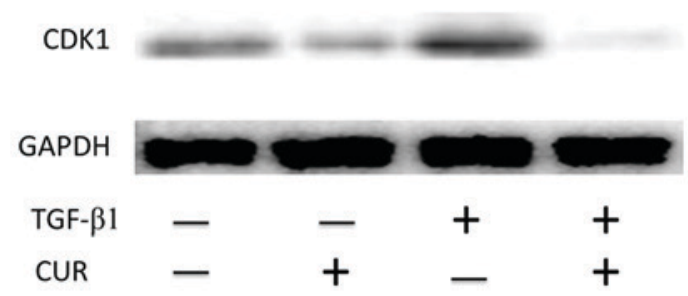

B

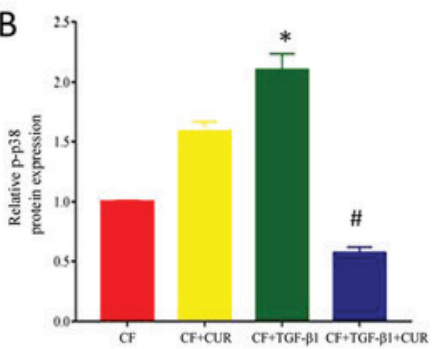

D

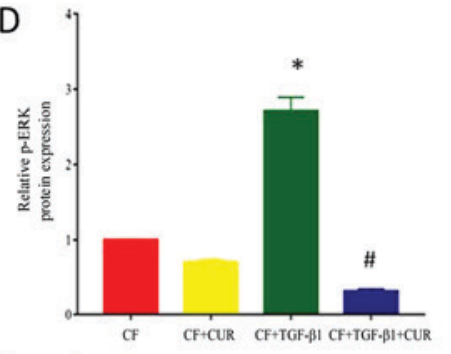

C

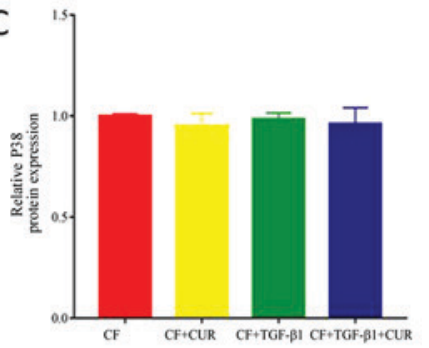

E

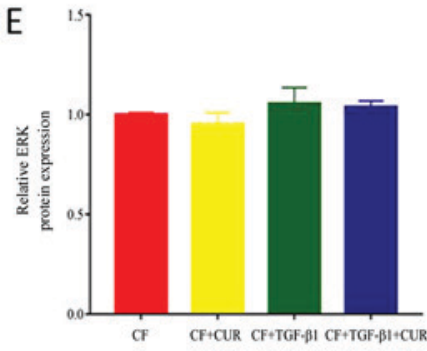

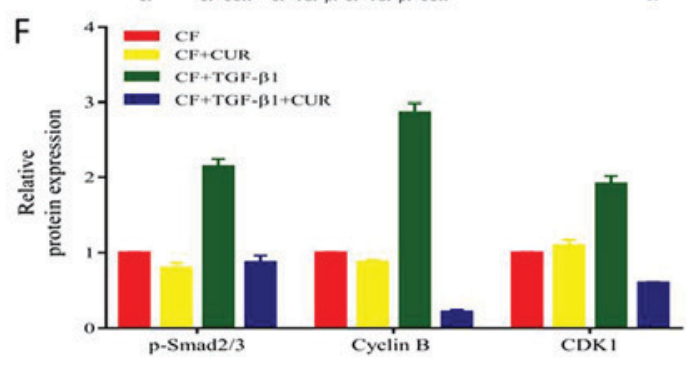

Figure 4. CUR affects cell-cycle protein expression by inhibiting p38 MAPK signaling activation in TGF- $\beta 1$-induced CFs. (A) Western blotting analysis of p-smad2/3, P38, p-P38, ERK, p-ERK, Cyclin B, and CDK1 expression. Quantitative analysis of (B) p-P38 protein expression using P38 as a control, (C) P38 protein expression using GAPDH as a control, (D) p-ERK protein expression using ERK as a control, (E) ERK protein expression using GAPDH as a control, and $(\mathrm{F})$ p-smad2/3, Cyclin B and CDK1 protein expression using GAPDH as a control. Data are presented as the mean \pm standard deviation of triplicate experiments. "P<0.05 vs. CF group; ${ }^{\#} \mathrm{P}<0.05$ vs. $\mathrm{CF}+\mathrm{TGF}-\beta 1$ group. CUR, curcumin; MAPK, mitogen-activated protein kinase; TGF, transforming growth factor; CFs, cardiac fibroblasts; ERK, extracellular signal-regulated kinase; p-, phosphorylated; CDK, cyclin dependent kinase; smad2/3, phosphorylation-mothers against decapentaplegic homolog $2 / 3$.

differentiation. Moreover, TGF- $\beta 1$ stimulation increased protein levels of collagen I and III; however, these effects were suppressed by curcumin. Taken together, these findings suggested that curcumin attenuated cardiac fibrosis by inhibiting myofibroblast differentiation, which is consistent with the findings presented in previous studies $(13,18)$.

Abnormal proliferation of cardiac fibroblast to myofibroblasts causes excessive synthesis and accumulation of extracellular matrix proteins, including collagen I and III, eventually leading to cardiac fibrosis. Suppression of proliferation and induction of apoptosis could help reduce activated fibroblasts and thus alleviate profibrogenic effects. In the present study, we demonstrated that curcumin regulated proliferation and the cell cycle phase of CFs in vitro. Our findings demonstrated that curcumin inhibited proliferation and promoted $\mathrm{G} 2 / \mathrm{M}$ phase arrest in TGF- $\beta 1$-induces CFs, which constituted one likely explanation of the cardiac protective effects of curcumin on cardiac fibrosis. Similarly, cyclin B, and CDK1, key modulators in G2/M checkpoint (19), were suppressed by curcumin treatment. In addition, curcumin had no effect on proliferation and cell cycle arrest in CFs that were not treated with TGF- $\beta 1$, indicating the safety in normal human CFs.

TGF- $\beta 1$ is a potent pro-fibrotic cytokine that acts through the canonical Smad pathway (20). After activation, p-Smad2 and 3 translocate into the nucleus to modulate the expression of downstream genes, thereby exerting pro-fibrotic effects (4).
In the present study, exogenous treatment with of CFs with TGF- $\beta 1$ significantly enhanced phosphorylation of Smad2/3, and this effect was significantly inhibited after administration of curcumin, which was in line with the findings of a previous study (21). In addition, TGF- $\beta 1$ can also act through non-canonical pathways, such as the MAPK c-Jun N-terminal kinase (c-JNK), ERK, and p38 MAPK pathways $(22,23)$. In this study, TGF- $\beta 1$ promoted p38 MAPK and p-ERK phosphorylation, while curcumin pretreatment markedly inhibited the increased phosphorylation of p38 MAPK, and p-ERK. These results were consistent with the data presented in a previous study (14). Taken together, these results suggested that curcumin inhibited p38 MAPK and p-ERK phosphorylation in TGF- $\beta 1$-stimulated CFs.

In conclusion, curcumin inhibited TGF- $\beta 1$-mediated CF activities, including differentiation and collagen deposition by inhibiting cell proliferation and promoting $\mathrm{G} 2 / \mathrm{M}$ phase arrest. Moreover, curcumin reduced cyclin B and CDK1 expression by inhibiting smad2/3, p38 MAPK, and ERK signaling activation in TGF- $\beta 1$-induced CFs. Thus, curcumin presents a promising drug for the treatment of cardiac fibrosis.

\section{Acknowledgements}

The authors would like to thank the Cardiac Surgery Laboratory for their technical assistance. 


\section{Funding}

No funding was received.

\section{Availability of data and materials}

The datasets used and/or analyzed during the current study are available from the corresponding author on reasonable request.

\section{Authors' contributions}

DL and GF conceived and designed the study. GF, SC, QH and LC performed the experiments and were major contributors in writing the manuscript. All authors read and approved the final manuscript.

\section{Ethics approval and consent to participate}

Not applicable.

\section{Consent for publication}

Not applicable.

\section{Competing interests}

The authors declare that they have no competing interests.

\section{References}

1. Schelbert EB, Piehler KM, Zareba KM, Moon JC, Ugander M, Messroghli DR, Valeti US, Chang CC, Shroff SG, Diez J, et al: Myocardial fibrosis quantified by extracellular volume is associated with subsequent hospitalization for heart failure, death, or both across the spectrum of ejection fraction and heart failure stage. J Am Heart Assoc 4: pii: e002613, 2015.

2. Kong P, Christia P and Frangogiannis N: The pathogenesis of cardiac fibrosis. Cell Mol Life Sci 71: 549-574, 2014.

3. Krenning G, Zeisberg E and Kalluri R: The origin of fibroblasts and mechanism of cardiac fibrosis. J Cell Physiol 225: 631-637, 2010.

4. Khan R and Sheppard R: Fibrosis in heart disease: Understanding the role of transforming growth factor-beta in cardiomyopathy, valvular disease and arrhythmia. Immunology 118: 10-24, 2006.

5. Ivey MJ and Tallquist MD: Defining the cardiac fibroblast. Circ J 80: 2269-2276, 2016.

6. Hewlings SJ and Kalman DS: Curcumin: A review of its' effects on human health. Foods 6: pii: E92, 2017.

7. Hazarey VK, Sakrikar AR and Ganvir SM: Efficacy of curcumin in the treatment for oral submucous fibrosis-A randomized clinical trial. J Oral Maxillofac Pathol 19: 145-152, 2015.
8. Chen YN, Hsu SL, Liao MY, Liu YT, Lai CH, Chen JF, Nguyen MT, Su YH, Chen ST and Wu LC: Ameliorative effect of curcumin-encapsulated hyaluronic acid-PLA nanoparticles on thioacetamide-induced murine hepatic fibrosis. Int J Environ Res Public Health 14: pii: E11, 2016.

9. Tyagi N, Dash D and Singh R: Curcumin inhibits paraquat induced lung inflammation and fibrosis by extracellular matrix modifications in mouse model. Inflammopharmacology 24: 335-345, 2016.

10. Hu Y, Mou L, Yang F, Tu H and Lin W: Curcumin attenuates cyclosporine A-induced renal fibrosis by inhibiting hypermethylation of the klotho promoter. Mol Med Rep 14: 3229-3236, 2016.

11. Yu W, Wu J, Cai F, Xiang J, Zha W, Fan D, Guo S, Ming Z and Liu C: Curcumin alleviates diabetic cardiomyopathy in experimental diabetic rats. PLoS One 7: e52013, 2012.

12. Wang NP, Wang ZF, Tootle S, Philip T and Zhao ZQ: Curcumin promotes cardiac repair and ameliorates cardiac dysfunction following myocardial infarction. Br J Pharmacol 167: 1550-1562, 2012.

13. Meng Z, Yu XH, Chen J, Li L and Li S: Curcumin attenuates cardiac fibrosis in spontaneously hypertensive rats through PPAR- $\gamma$ activation. Acta Pharmacol Sin 35: 1247-1256, 2014.

14. Chung CC, Kao YH, Liou JP and Chen YJ: Curcumin suppress cardiac fibroblasts activities by regulating proliferation, migration, and the extracellular matrix. Acta Cardiol Sin 30: 474-482, 2014.

15. Porter KE and Turner NA: Cardiac fibroblasts: At the heart of myocardial remodeling. Pharmacol Ther 123: 255-278, 2009.

16. van den Borne SW, Diez J, Blankesteijn WM, Verjans J, Hofstra L and Narula J: Myocardial remodeling after infarction: The role of myofibroblasts. Nat Rev Cardiol 7: 30-37, 2010.

17. de Haas HJ, Arbustini E, Fuster V, Kramer CM and Narula J: Molecular imaging of the cardiac extracellular matrix. Circ Res 114: 903-915, 2014.

18. Li HL, Liu C, de Couto G, Ouzounian M, Sun M, Wang AB Huang Y, He CW, Shi Y, Chen X, et al: Curcumin prevents and reverses murine cardiac hypertrophy. J Clin Invest 118: 879-893, 2008.

19. Canaud G and Bonventre JV: Cell cycle arrest and the evolution of chronic kidney disease from acute kidney injury. Nephrol Dial Transplant 30: 575-583, 2015.

20. Derynck R and Zhang YE: Smad-dependent and Smad-independent pathways in TGF-beta family signalling. Nature 425: 577-584, 2003.

21. Ma J, Ma S and Ding CH: Curcumin reduces cardiac fibrosis by inhibiting myofibroblast differentiation and decreasing transforming growth factor $\beta 1$ and matrix metalloproteinase $9 /$ tissue inhibitor of metalloproteinase 1. Chin J Integr Med 23: 362-369, 2017.

22. Voloshenyuk TG, Landesman ES, Khoutorova E, Hart AD and Gardner JD: Induction of cardiac fibroblast lysyl oxidase by TGF- $\beta 1$ requires PI3 K/Akt, Smad3, and MAPK signaling. Cytokine 55: 90-97, 2011.

23. Zhang D, Liu X, Chen X, Gu J, Li F, Zhang W and Zheng Y: Role of the MAPKs/TGF- $\beta 1 /$ TRAF6 signaling pathway in atrial fibrosis of patients with chronic atrial fibrillation and rheumatic mitral valve disease. Cardiology 129: 216-223, 2014.

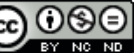

This work is licensed under a Creative Commons Attribution-NonCommercial-NoDerivatives 4.0 International (CC BY-NC-ND 4.0) License. 